\title{
Adsorption-induced constraint on delocalization of electron states in an Au chain on $\operatorname{NiAl}(110)$
}

\author{
Mats Persson* \\ Department of Applied Physics, Chalmers/Göteborgs University, S-41296, Göteborg, Sweden
}

(Dated: September 17, 2018)

\begin{abstract}
We have carried out a density functional study of the localized constraint on the delocalized, unoccupied resonance states in a mono atomic Au chain on a $\mathrm{NiAl}(110)$ surface by adsorption of a $\mathrm{CO}$ molecule on one of the adatoms in the chain. This constraint was observed recently by scanning tunnelling microscopy and spectroscopy. The repulsive interaction of the occupied $5 \sigma$ molecular state with the unoccupied, resonance state in a $\mathrm{Au}$ adatom in the chain is found to break the degeneracy of the Au adatom-induced resonance states and to disrupt their delocalization in the chain.

PACS numbers: 73.20.Hb, 73.21.Hb, 68.37.Ef
\end{abstract}

Adsorbate-induced modifications of confined electronic states in metallic nanostructures on surfaces such as supported atomic wires are of direct interest in chemical sensing and also in the emerging field of molecular electronics through their influence on electron transport 1.2.3.4.5. To gain physical insight about these complex phenomena, one needs to study how the confined electronic states of a single, adatom nanostructure with well-defined atomic structure and composition is affected by controlled adsorption of a single atom or molecule. This goal was recently realized by a scanning tunnelling spectroscopy study of fabricated monoatomic Au atom chains of various lengths on a $\mathrm{NiAl}(110)$ surface upon adsorption of a single $\mathrm{CO}$ molecule at various sites along the chain ${ }^{6}$. The bare chains have been shown to support "particle-in-box"-like unoccupied electron states? tially resolved measurements of these electronic states revealed that the adsorbed $\mathrm{CO}$ molecule introduced a localized constraint on the electron delocalization in the chain. Thus there is a need to understand the physics behind this adsorption-induced constraint from a theoretical study of these electronic states.

The electronic structure of monoatomic Au adatom chains on a $\mathrm{NiAl}(110)$ surface has been studied recently by density functional calculations ${ }^{8.9}$. In particular, the study in Ref. 9 have revealed the nature of the unoccupied, resonant states in these chains and their "particlein-box" character. The observed energy splittings of the resonance doublets of $\mathrm{Au}$ addimers as a function of the interatomic distance $\frac{10}{}$ and the observed energy dispersion of the resonance states in Au chains were well reproduced by the calculated, unoccupied Kohn-Sham states 11 in this study. The resonance states were shown to derive from the $6 s$ states of the $\mathrm{Au}$ atoms, which develop a $p_{z}$ character upon adsorption. The resonance states were found to interact strongly with the substrate states as reflected by long-range, substrate-mediated interactions between adatom resonances. In contrast, Calzolari and coworkers ${ }^{8}$ argued from their density functional study of transport in $\mathrm{Au}$ ad-chains that they behaved as isolated $\mathrm{Au}$ chains. They also studied the electronic and geometric structure of adsorption of a $\mathrm{CO}$ molecule on a seven
$\mathrm{Au}$ adatom long chain. A repulsive interaction induced by the $\mathrm{Au}-\mathrm{C}$ bond was found to induce a interaction that pushed the $\mathrm{Au} 6 s$ level to higher energies. Because they did not identify any unoccupied resonance states of the adsorbed $\mathrm{Au}$ chain, it is not clear how this repulsion results in a localized constraint on the delocalization of these states in the adsorbed chain.

In this paper, we report a density functional study of the influence of an adsorbed $\mathrm{CO}$ molecule on the electronic states of an $\mathrm{Au}$ adatom and adtrimer on a $\mathrm{NiAl}(110)$ surface. These adatom structures albeit being of minimal size reveal directly the physics behind the localized constraint on the delocalization of the unoccupied electron states. This physics can be directly generalized to $\mathrm{Au}$ adatom chains of arbitrary lengths.

In order to reveal the electronic and geometric effects of an adsorbed $\mathrm{CO}$ molecule on mono-atomic Au chains on $\mathrm{NiAl}(110)$, we have carried out density functional calculations of $\mathrm{CO}$ molecules adsorbed on an $\mathrm{Au}$ adatom and on an edge and center adatom of an $\mathrm{Au}$ ad-trimer. As in the previous study of the $\mathrm{Au}$ adatom and ad-trimer ${ }^{9}$, these calculations were carried out using the projector augmented wave method as implemented in the VASP code $\frac{12}{2}$. The exchange and correlation effects were represented by the PW91 version ${ }^{13}$ of the generalized gradient approximation. The systems were represented by a $\mathrm{CO}$ molecule and $\mathrm{Au}$ atoms on a NiAl slab in a super cell geometry ${ }^{14}$ and the equilibrium structures were obtained by structural optimization ${ }^{15}$. The nature of the electronic states was investigated by calculating the partial wave decomposition of the density of states within atomic spheres 16 and the local density of states $\frac{17}{}$ outside the surface.

The calculated equilibrium structures of a CO molecule on a single $\mathrm{Au}$ adatom and $\mathrm{Au}$ ad-trimer are depicted in Fig. 1] The equilibrium structures of the bare single $\mathrm{Au}$ adatom and ad-trimer were already discussed in an earlier density functional study ${ }^{9}$ and are not discussed here. The $\mathrm{CO}$ molecule is adsorbed in an upright position on a single $\mathrm{Au}$ adatom and on the center atom of the $\mathrm{Au}$ ad-trimer and in a slightly tilted configuration on an edge atom of the $\mathrm{Au}$ ad-trimer 18 . The adsorption of the $\mathrm{CO}$ molecule on the edge atom is only about $0.023 \mathrm{eV}$ more 

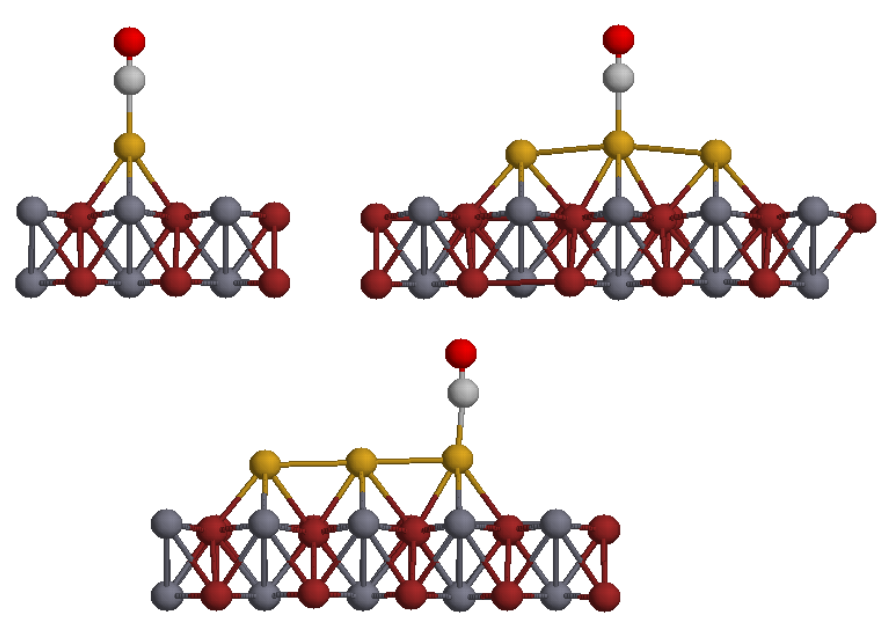

FIG. 1: Ball and stick model of the calculated geometric structure of an $\mathrm{CO}$ molecule adsorbed on (A) a single $\mathrm{Au}$ adatom, and $(\mathrm{B})$ the center adatom and $(\mathrm{C})$ the edge adatom of an $\mathrm{Au}$ ad-trimer. Only the two outermost layers of the $\mathrm{NiAl}(110)$ substrate are shown.

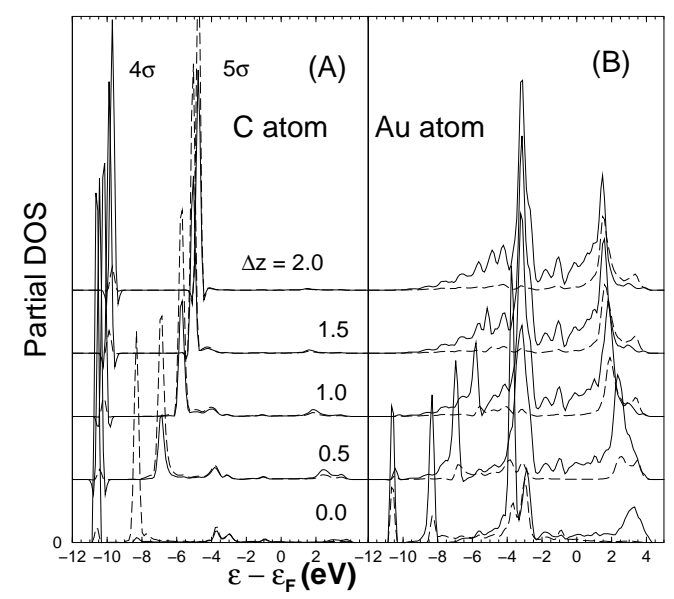

FIG. 2: Evolution of calculated partial density of $s$ (solid lines) and $p_{z}$ (dashed lines) states within a sphere around (A) a $\mathrm{C}$ atom and (B) a $\mathrm{Au}$ atom in $\mathrm{CO} / \mathrm{Au} / \mathrm{NiAl}(110)$ as a function of distance $\delta z$ of the $\mathrm{CO}$ molecule from its equilibrium position on the $\mathrm{Au}$ adatom. The peaks in the partial DOS for the $\mathrm{C}$ atom derive from the $4 \sigma$ and $5 \sigma$ molecular orbitals of the $\mathrm{CO}$ molecule as indicated.

stable than the adsorption site on the center atom. This finding is consistent with the experimental observation that tunnelling at high bias and current tended tended to displace the $\mathrm{CO}$ admolecule from the interior to the ends of the chains ${ }^{6}$. The main effect of the $\mathrm{CO}$ adsorption on the $\mathrm{Au}$ adatoms is an upward shift of the $\mathrm{Au}$ adatom coordinated to the $\mathrm{O}$ atom by about $0.1 \AA$ for the center adsorption site on the $\mathrm{Au}$ ad-trimer and by about $0.2 \AA$ for the other two structures.

The effect of the adsorption of a $\mathrm{CO}$ molecule on a single $\mathrm{Au}$ adatom on its unoccupied, resonance state $6 \tilde{\sigma}$ is revealed by studying the evolution of the partial DOS around the $\mathrm{Au}$ adatom (Fig. 22A) and the $\mathrm{C}$ atom
(Fig. 20 B) as a function of the $\mathrm{CO}$ molecule distance, $\delta z$, from the $\mathrm{Au}$ adatom. At $\delta z=2 \AA$, the electron states of the $\mathrm{CO}$ molecule and the $\mathrm{Au}$ adatom are essentially decoupled. The $6 \tilde{\sigma}$ state of the Au adatom show up as a relatively narrow peak at about $1.6 \mathrm{eV}$ above the Fermi level in the DOS of $s$ and $p_{z}$ partial waves around the $\mathrm{Au}$ adatom. The partial DOS with $s$ character covers a wide energy range and exhibits also a relatively narrow resonance structure at $3.5 \mathrm{eV}$ below the Fermi level. The DOS for $s$ and $p_{z}$ partial waves around the $\mathrm{C}$ atom are dominated by the $4 \sigma$ and $5 \sigma$ molecular orbitals of the $\mathrm{CO}$ molecule. Around the $\mathrm{C}$ atom, $4 \sigma$ has predominantly $s$ character, whereas $5 \sigma$ has predominantly $p_{z}$ character. The $1 \pi$ and the $2 \pi$ molecular orbitals of the CO molecule are symmetry forbidden to couple with the $6 \tilde{\sigma}$ state and are not shown in Fig 2AA.

At smaller $\delta z$, the $5 \sigma$ state starts to interact with the $6 \tilde{\sigma}$ state, resulting in a repulsion between these two states and an interchange of their characters. The $6 \tilde{\sigma}$ state shifts up with about $1.5 \mathrm{eV}$ and the $5 \sigma$ state shifts down with about $3 \mathrm{eV}$. In contrast, the $4 \sigma$ state shows only a minor downward shift in energy. Note that the strong interaction between the $5 \sigma$ state and the $6 \tilde{\sigma}$ state is manifested by the repulsion of about $4.5 \mathrm{eV}$ between these two states being substantial in comparison between their original energy separation of about $6 \mathrm{eV}$. The $5 \sigma$ state keeps its $p_{z}$ character on the $\mathrm{C}$ atom but loses its $s$ character and develops an admixture of predominantly $s$ character on the $\mathrm{Au}$ adatom. In contrast, the $6 \tilde{\sigma}$ state keeps its $s$ character on the $\mathrm{Au}$ adatom but loses its $p_{z}$ character. The resonance structure around $-3.5 \mathrm{eV}$ in the $s$ DOS around the Au adatom is weakly affected by the interaction with the $\mathrm{CO}$ molecule.

In the Au adchains, the degenerate $6 \tilde{\sigma}$ states of the individual adatoms interact and form delocalized resonant states. The upward energy shift of the $6 \tilde{\sigma}$ state of an $\mathrm{Au}$ adatom by the $\mathrm{CO}$ adsorption on the $\mathrm{Au}$ chains breaks its degeneracy with the other $6 \tilde{\sigma}$ states in the chain and has important consequences on the delocalization of the $6 \tilde{\sigma}$ states in the chain. These consequences are here illustrated for $\mathrm{CO}$ adsorption on the $\mathrm{Au}$ trimer on the $\mathrm{NiAl}(110)$ surface. First we need to describe the nature of resonance states of the bare adtrimer.

The interactions among the three degenerate adatom $6 \tilde{\sigma}$ states in the trimer results in a resonance triplet with large energy splittings as revealed by the calculated LDOS on-top of the center and edge adatoms in Fig. 3A. The first and the third state at 1.0 and $2.1 \mathrm{eV}$, respectively, are both symmetric where the former state has the largest amplitude on the center adatom and the latter state has its largest amplitude on the edge adatoms. The second state at $1.6 \mathrm{eV}$ is antisymmetric and has accordingly no amplitude on the center adatom. The interaction between the $6 \tilde{\sigma}$ states of the two edge adatoms is weak so that the energy of this state is close to the energy of $1.7 \mathrm{eV}$ for the $6 \tilde{\sigma}$ state in a single adatom. The energies of the first and third state are symmetrically split around this energy. 


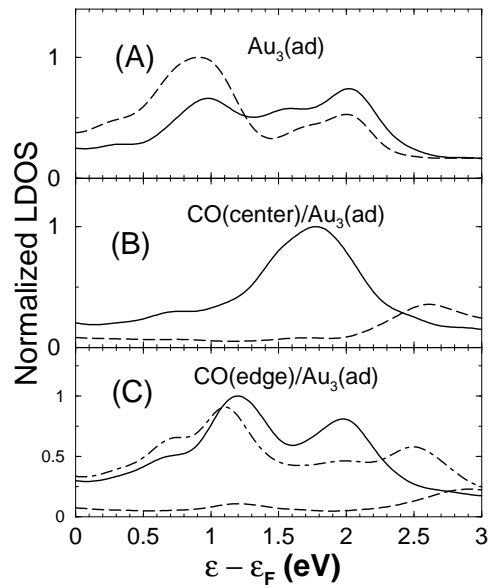

FIG. 3: Normalized local density of states (LDOS) for (A) $\mathrm{Au}$ ad-trimer and a $\mathrm{CO}$ molecule adsorbed on (B) the center adatom or (C) the edge adatom of the $\mathrm{Au}$ adtrimer. The LDOS has been calculated at a distance of about $1 \AA$ above an edge adatom (solid and dashed-dotted lines) and the center (dashed lines) adatom in the $\mathrm{Au}$ ad-trimer. In (C), the two edge atoms are inequivalent and the solid and the dasheddotted line gives LDOS above the edge atom without and with an adsorbed CO molecule, respectively. The LDOSs are normalized to their maximum values above the center adatom in $(\mathrm{A})$ and $(\mathrm{C})$, and the edge adatom in (B).

Upon adsorption of a $\mathrm{CO}$ molecule on an adatom in the adtrimer, the resonance structure of the adtrimer changes dramatically (Fig. 3). For the center adatom adsorption site, the resonance triplet collapses to a resonance singlet (Fig. 3B), whereas for the edge adatom it collapses to a resonance doublet (Fig. $3 \mathrm{C}$ ). The resonance singlet and doublet energies are very close to the values $1.7 \mathrm{eV}$ for the $6 \tilde{\sigma}$ state in a single adatom and 1.2 and $2.15 \mathrm{eV}$ for the resonance doublet in the $\mathrm{Au}$ addimer, respectively. The adsorption-induced breaking of the degeneracy of the $6 \tilde{\sigma}$ state in the adatom coordinated to the $\mathrm{CO}$ molecule with the $6 \tilde{\sigma}$ states of the remaining adatoms effectively turns off its interaction with these latter states. In the case of adsorption on the center adatom, the interaction between the $6 \tilde{\sigma}$ states of the two edge atoms, as mediated in the bare adtrimer essentially by the $6 \tilde{\sigma}$ state of the center adatom, is turned off. This results in essentially two degenerate and isolated resonance states. In the case of $\mathrm{CO}$ adsorption on an edge adatom, there is still a direct interaction between the two $6 \tilde{\sigma}$ states on the remaining two adatoms, which splits them into a doublet.

The calculated changes in the resonance energy structure of the adtrimer upon adsorption of a $\mathrm{CO}$ molecule can be rationalized in a simple, tight binding resonance model. This model was introduced in an earlier paper ${ }^{9}$ and applied to various linear Au adatom structures. The resonance triplet energies and states of the bare adtrimer can be modelled by a value of $1.6 \mathrm{eV}$ for the on-site energy $\epsilon_{0}$ for the adatoms and a value of $-0.35 \mathrm{eV}$ for the nearest-neighboring off-site energy $t$. Note that values of $\epsilon_{0}$ and $2|t|$ are close to the values for the calculated energy $1.7 \mathrm{eV}$ of the $6 \tilde{\sigma}$ state in the adatom and the calculated energy splitting $0.9 \mathrm{eV}$ of the resonance double in an addimer. In this model, the resulting triplet energies are given by $\epsilon_{0}+t \sqrt{2}=2.1, \epsilon_{0}=1.6$, and $\epsilon_{0}-t \sqrt{2}=1.1$ $\mathrm{eV}$ and are close the calculated resonance energies of 2.1, 1.6 and $1.0 \mathrm{eV}$. As suggested by the calculated upward energy shift of about $1.5 \mathrm{eV}$ for the $6 \tilde{\sigma}$ by $\mathrm{CO}$ adsorption on a single adatom, the effect of the corresponding adsorption of a $\mathrm{CO}$ molecule on an adatom in the adtrimer is then modelled by increasing the associated on-site energy by $\Delta \epsilon=1.5 \mathrm{eV}$. For simplicity, the off-site energies are assumed to be unaffected by the adsorption. The adsorption-induced effects on the electronic states of the ad-trimer is understood semi-quantitatively in this model by perturbation theory in the relatively small parameter $|t| / \Delta \epsilon$.

We apply this model first to the case of $\mathrm{CO}$ adsorption on the center adatom. To zeroth order in $t$, only the energy of the resonance state of the center adatom is shifted up by $\Delta \epsilon$. The degeneracy of the two resonance states of the edge atoms is split first to second order in $t$. To this order these states are split by $2|t|^{2} / \Delta \epsilon=0.16$ $\mathrm{eV}$ and both shift down by $2|t|^{2} / \Delta \epsilon=0.16 \mathrm{eV}$. Thus the energy of antisymmetric state in this resonance doublet is unperturbed and is the same as for the anti-symmetric state of the bare ad-trimer. This behavior reflects that the perturbation by adsorbed CO molecule is localized to the center adatom and does not break the symmetry of the ad-trimer. The energy splitting of the resonance doublet is small compared to the resonance width and is not resolved in the calculated LDOS so the doublet appears as a single broad resonance.

In contrast to $\mathrm{CO}$ adsorption on a center adatom the adsorption on an edge atom breaks the symmetry of the ad-trimer. The resonance energy of this edge atom is shifted up by $\Delta \epsilon$ and the degeneracy of the two resonance states at the center adatom and the bare edge adatom is split to leading order in $t$ by $2|t|$. The resulting resonance doublet has then the same energies as for the resonance doublet of the ad-dimer. The corrections to these energies by second order perturbations in $t$ are small. The resonance doublet energies shift down only by about $|t|^{2} /(2 \Delta \epsilon)=0.04 \mathrm{eV}$ and the effect on the energy splitting is of higher order in $t$.

In summary, we have carried out a density functional study of the effects on the delocalized, unoccupied resonance states in a mono atomic $\mathrm{Au}$ chain on a $\mathrm{NiAl}(110)$ surface upon adsorption of a $\mathrm{CO}$ molecule on one of the adatoms in the chain. This study explains the observed localized constraint by the adsorbed CO molecule on these states in terms of a repulsive interaction of the occupied $5 \sigma$ molecular state with the unoccupied, resonance state in the Au adatom coordinated to the adsorbed CO molecule. This interaction breaks the degeneracy of the $\mathrm{Au}$ adatom-induced resonance states in the chain and constrains their delocalization. This physical mechanism should be of more general interest and ap- 
ply whenever the adsorbed molecule has frontier orbitals that are symmetry allowed to interact with the atomic orbitals in atomic wires that participate in the formation of delocalized states.

\section{Acknowledgments}

Financial support from the Swedish Research Council (VR) and the Swedish Strategic Foundation (SSF) through the materials consortium ATOMICS, ISIS, UC Irvine and allocation of computer resources through SNAC are gratefully acknowledged. The author is indebted to N. Nilius, W. Ho, D. L. Mills, and R. B. Muniz for many stimulating discussions and additional insights.
* Electronic address: tfymp@fy.chalmers.se

1 D. R. Bowler, J. Phys.: Condens. Matter 16, R721 (2004).

2 J. Kong et al., Science 287, 622 (2000).

3 P. G. Collins, et al., Science 287, 1801 (2000).

${ }^{4}$ F. Favier, et al., Science 293, 2227 (2000).

${ }^{5}$ H. E. Romero, K. Bolton, A. Rosén, and P. C. Eklund, Science 307, 89 (2005).

6 N. Nilius, T. M. Wallis, and W. Ho, Phys. Rev. Lett. 90, 186102 (2003).

7 N. Nilius, T. M. Wallis, and W. Ho, Science 297, 1853 (2002).

8 A. Calzolari, C. Cavazzoni, and M. B. Nardelli, Phys. Rev. Lett. 93, 096404 (2004).

9 M. Persson, Phys. Rev. B 70, 205420 (2004).

10 N. Nilius, T. M. Wallis, M. Persson, and W. Ho., Phys. Rev. Lett. 90, 196103 (2003).

11 In principle, the unoccupied, resonance states of the $\mathrm{Au}$ adatom structures are probed by adding an electron and cannot be directly described by conventional density functional theory.

12 G. Kresse and J. Hafner, Phys. Rev. B47, 558 (1993); G. Kresse and J. Furthmüller, Phys. Rev. B54, 11169 (1996);
G. Kresse and J. Joubert, Phys. Rev. B59, 1758 (1999).

13 J. P. Perdew et al., Phys. Rev. B 46, 6671 (1992).

14 For the $\mathrm{CO}$ adsorption on the $\mathrm{Au}$ adatom and the adtrimer, we used a $3 \times 2$ and $5 \times 2$ surface unit cell, respectively, and $8 \mathrm{NiAl}$ layers in the slab. To accommodate the adsorbed $\mathrm{CO}$ molecule, we used a vacuum region of about $14.4 \AA$. The kinetic energy cut off of the plane wave basis set was about $300 \mathrm{eV}$ and the surface Brillouin zones for the $3 \times 2$ and $5 \times 2$ surface unit cells were sampled uniformly by $6 \times 6$ and $4 \times 6 k$ points, respectively.

15 The $\mathrm{C}, \mathrm{O}, \mathrm{Au}$ atoms and the two outermost NiAl layers were fully relaxed until the residual forces were less than $0.05 \mathrm{eV} / \AA$.

16 The atomic sphere radi were chosen to be $0.73,0.77,1.45$, 1.25 , and $1.43 \AA$ for the $\mathrm{O}, \mathrm{C}, \mathrm{Au}, \mathrm{Ni}$ and $\mathrm{Al}$ atoms, respectively.

17 D. R. Hamann, and J. Tersoff, Phys. Rev. Lett. 50, 1998 (1983).

18 The C-O bond length is about $1.15 \AA$ upon adsorption and the O-Au bond length is 1.99 and $1.98 \AA$ for structures (A) and (B), and for structure (C), respectively, in Fig. 1] 